\title{
Isolation of Aspergillus species in respiratory specimens from adult patients with acute exacerbation of non-cystic fibrosis bronchiectasis; Data from Pakistan
}

\author{
Jabeen $\mathrm{K}^{\mathbf{1}}$, Irfan $\mathbf{M}^{\mathbf{2}}$, Khan $\mathbf{M}^{\mathbf{1}}$, Farooqi JQ1', Iqbal $\mathbf{N}^{\mathbf{2}}$ \\ 1 Department of Pathology and Laboratory Medicine, Aga Khan University, Karachi, Pakistan \\ 2 Section of Pulmonary and Critical care, Department of Medicine, Aga Khan University, Karachi, Pakistan
}

\section{Background:}

Bronchiectasis unrelated to cystic fibrosis (Non-CF) is increasingly being recognized as a chronic respiratory illness in developing countries. Patients present with frequent respiratory infections and exacerbation of their illness. In such patients role of fungal isolation during these episodes in not clear ${ }^{1}$. Limited evidence suggests that isolation and persistence of fungi are frequent in patients with non-CF especially in patients with prior antibiotic therapy ${ }^{2}$. Although non-CF bronchiectasis is common in Pakistan, no data exists regarding the spectrum of fungi isolated from the bronchiectasis patient population during an episode of exacerbation.

\section{Objectives:}

To determine isolation rate of Aspergillus species in respiratory specimens from adult patients with acute exacerbation of noncystic fibrosis bronchiectasis presenting to Aga Khan University, Hospital (AKUH), Karachi, Pakistan

\section{Methods:}

This cross sectional study (2015-2017) was performed at AKUH, Karachi, Pakistan. Clinical and laboratory details of adult patients presenting to AKUH pulmonology clinics with acute exacerbation of non CF bronchiectasis was retrieved from medical records. Microbial cultures (bacterial, fungal and mycobacterial) were performed in the AKU laboratory. All specimens were processed and reported using American Society of Microbiology standards

Ethical Approval: Study was approved from the ERC of the Aga Khan University
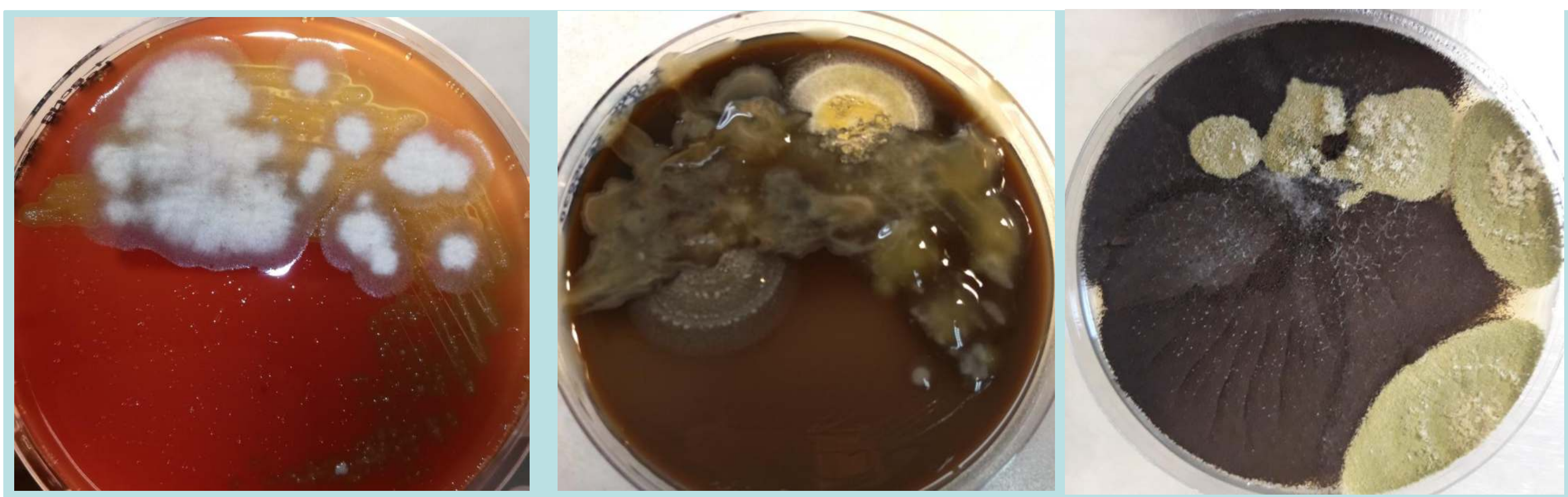

Images show mold as the only pathogen (left), mixed with bacterial pathogens (center) and multiple molds in the same sample (right)

\section{Results:}

- Of the 338 non-cystic fibrosis bronchiectasis patients presenting to $\mathrm{AKUH}, 206$ patients submitted 437 sputum samples

- Aspergillus species were isolated from 27/206 (13\%) patients

- Predominant species was A. flavus (16 patients), A. niger (11 patients) and $A$. fumigatus (8 patients)

- In 60 patients where clinicians specifically requested for fungal culture Aspergillus species isolation rate was 18/60 (30\%) higher as opposed to 9/144 (6\%) in patients where Aspergillus species was isolated from routine bacterial culture

\section{Spectrum of fungal isolation $(n=27)$}

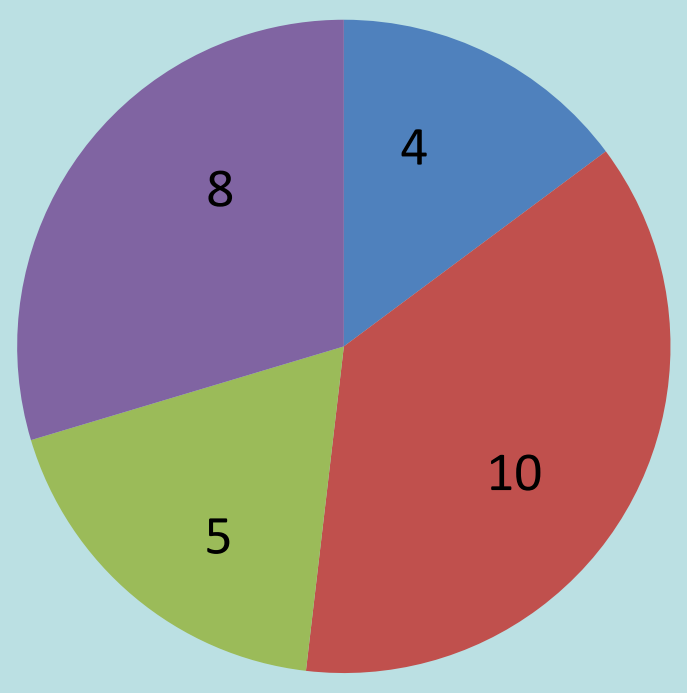

- Single Aspergillus spp.

Polymicrobial with Pseudomonas aeruginosa

Polymicrobial with Hemophilus influenzae

\section{Conclusion:}

Despite of variability in processing of respiratory specimens cultures, Aspergillus species was isolated in significant proportion of patients presenting with acute exacerbation of non-cystic fibrosis bronchiectasis at AKUH. Clinical significance needs to be assessed by prospective evaluation using standardized microbiological methods, Aspergillus specific serological tests and appropriate treatment regimens 